\title{
Spin-orbit coupling mediated spin torque in a single ferromagnetic layer
}

\author{
A. Matos-Abiague \\ Institute for Theoretical Physics, University of Regensburg, 93040 Regensburg, Germany \\ R. L. Rodríguez-Suárez \\ Departamento de Física, Pontifícia Universidad Católica de Chile, Casilla 306, Santiago 6904411, Chile
}

(Received 25 May 2009; revised manuscript received 13 August 2009; published 30 September 2009)

\begin{abstract}
By considering a linear in momentum but otherwise arbitrary spin-orbit coupling (SOC), we derive a simple analytical expression for the current-driven spin torque in a single ferromagnetic layer. Explicit forms of the spin torque are given for structures with different SOC fields, in dependence of strain effects, growth direction, and/or symmetry under spatial inversion. The Landau-Lifshitz-Gilbert equation including the effects of the SOC mediated spin torque on the magnetization dynamics is briefly discussed.
\end{abstract}

DOI: 10.1103/PhysRevB.80.094424

PACS number(s): 75.60.Jk, 75.75.+a, 72.25.-b, 72.10.-d

\section{INTRODUCTION}

Since its first theoretical description ${ }^{1,2}$ the phenomenon of spin-transfer torque has attracted increasing attention due to its potential applications in spintronic devices. This phenomenon occurs in spin-valve structures composed of two ferromagnetic layers separated by a nonmagnetic one. A transverse (perpendicular to the layers) charge current through the device produces a flow of spin-polarized conduction electrons from the fixed layer into the free layer. This causes a direct transfer of angular momentum from the spin-polarized flowing electrons to the local magnetization of the free layer, ${ }^{1-7}$ resulting in a torque that may produce magnetic reversal or steady-state precessions with frequencies in the microwave range. ${ }^{7-14}$ This spin-transfer mechanism allows nanomagnets to be manipulated without magnetic fields, and is the subject of extensive research for new applications in nonvolatile memory technology and radio-frequency oscillators.

Up to now the majority of investigations on the spin transfer driven excitations have been performed on planar spinvalve nanopillar or nanocontact structures in which a noncollinear configuration of the magnetic structure is required. However, spin torque phenomena may also be present in collinear spin valves composed of two ferromagnetic contacts separated by a two-dimensional electron gas with spinorbit coupling (SOC) ${ }^{15}$ Recent theoretical investigations ${ }^{16,17}$ have shown that, even in a single, uniformly magnetized ferromagnetic layer, when SOC is present, an in-plane current can induce a spin torque on the magnetization of the layer without the need for noncollinear ferromagnetic configuration of the structure. In these previous studies ${ }^{16,17}$ the authors considered a (001) ferromagnetic layer with magnetization lying on the plane of the layer and in the presence of Bychkov-Rashba ${ }^{18}$ and Dresselhaus ${ }^{19}$ SOCs. Here we investigate the current-driven SOC mediated spin torque for the case of an arbitrary magnetization orientation and a linear in momentum but otherwise arbitrary form of the SOC.

The paper is organized as follows. In Sec. II we present the basic assumptions and theoretical model. A general expression for the SOC mediated spin torque is derived in Sec. III for the case of a single, uniformly magnetizated ferromagnetic layer in the presence of a linear in momentum SOC field. The modified Landau-Lifshitz-Gilbert (LLG) equation accounting for the SOC mediated torque is briefly discussed in Sec. IV. Specific expressions for the SOC mediated spin torque are given in Sec. V for the cases of (001), (110), and (111) ferromagnetic layers with structure inversion asymmetry (SIA) and/or bulk inversion asymmetry (BIA). The spin torque mediated by strain-induced SOC is investigated in Sec. VI. Finally, conclusions are given in Sec. VII.

\section{THEORETICAL MODEL}

We consider a two-dimensional uniformly magnetized ferromagnetic layer and assume a model in which the local magnetization of the ferromagnet is determined by localized $d$-like electrons rather than from itinerant electrons. Such a model appears to be appropriate for electrodes composed of $3 d$ ferromagnetic metals and alloys lying on the negative slope side of the Slater-Pauling curve (e.g., $\mathrm{Co}, \mathrm{CoFe}, \mathrm{Ni}$, and $\mathrm{NiFe}) .6,20,21$

The localized spins couple to the itinerant spins through the exchange interaction

$$
H_{e x}=-J_{e x} \sum_{i, j}\left(\mathbf{S}_{i} \cdot \mathbf{s}_{j}\right),
$$

where $J_{e x}$ is the exchange coupling constant, $\mathbf{S}_{i}$ denotes the localized spin at the $i$ th site and $\mathbf{s}_{j}$ is the spin of the $j$ th itinerant electron.

Averaging Eq. (1) over the localized states, summing up the contributions of all the localized spins, and considering

$$
\mathbf{M}=\gamma_{0}\left\langle\left\langle\mathbf{S}_{i}\right\rangle\right\rangle_{\mathrm{loc}}=\gamma_{0} \sum_{i}\left\langle\mathbf{S}_{i}\right\rangle,
$$

with $\mathbf{M}, \gamma_{0}$, and $\left\langle\mathbf{S}_{i}\right\rangle$ as the macroscopic magnetization, gyromagnetic factor, and local spin density at site $i$, respectively, one obtains the effective one-particle exchange Hamiltonian for an itinerant electron,

$$
H_{e x}^{\mathrm{it}}=-\frac{J_{e x}}{\gamma_{0}}(\mathbf{M} \cdot \mathbf{s}),
$$

where $\hat{\mathbf{M}}=\mathbf{M} / M_{s}$ (with $M_{s}$ as the saturation magnetization) is a unit vector along the magnetization direction. Equation 
(3) can be rewritten in the more familiar form

$$
H_{e x}^{\mathrm{it}}=-\Delta_{e x}(\hat{\mathbf{M}} \cdot \boldsymbol{\sigma})
$$

by introducing the exchange splitting energy as

$$
\Delta_{e x}=\frac{J_{e x} \hbar M_{s}}{2 \gamma_{0}} \text {. }
$$

Thus, in the presence of SOC, the motion of the itinerant electrons is described by the one-particle Hamiltonian

$$
H^{\mathrm{it}}=\frac{\hbar^{2} k^{2}}{2 m}-\Delta_{e x} \hat{\mathbf{M}} \cdot \boldsymbol{\sigma}+\mathbf{w}(\mathbf{k}) \cdot \boldsymbol{\sigma},
$$

where $k$ is the length of the in-plane wave vector $\mathbf{k}, m$ is the carrier mass, and $\boldsymbol{\sigma}$ is a vector whose components are the Pauli matrices. The last term in Eq. (6) represents the SOC which is determined by the SOC field (SOCF) $\mathbf{w}(\mathbf{k})$. The time-reversal symmetry implies that $\mathbf{w}(\mathbf{k})=-\mathbf{w}(-\mathbf{k})$. Therefore, in the lowest approximation, the SOCF is always linear in the wave vector. One can then express the $i$ th component of the SOCF as

$$
w_{i}=\sum_{l=x, y} c_{i}^{l} k_{l} ; \quad(i=x, y, z)
$$

where $k_{l}$ are the wave-vector components and the coefficients $c_{i}^{l}$ determine the explicit form of the SOCF. Corrections of higher order in $\mathbf{k}$ may, in principle, play some role when large currents are applied and states with high values of $k_{\|}$become relevant. Furthermore, it has already been shown that up to the first order in $\Delta_{e x} / w_{i}\left(k_{F}\right)$ (with $k_{F}$ as the length of the Fermi wave vector) the cubic terms in the SOCF do not contribute to the torque. ${ }^{17}$ Here we consider the case of a strong ferromagnet for which $\Delta_{e x} \ll \mathbf{w}\left(\mathbf{k}_{\mathbf{F}}\right)$ and limit our analysis to the case of moderate currents. In such a regime, the linear approximation used in Eq. (7) suffices.

The effective exchange Hamiltonian for the localized spins can be obtained from Eq. (1) by averaging over the ensemble of itinerant electrons. The result is

$$
H_{e x}^{\mathrm{loc}}=-J_{e x}(\mathbf{S} \cdot\langle\langle\mathbf{s}\rangle\rangle),
$$

where $\langle\langle\mathbf{s}\rangle\rangle$ is the spin density of itinerant electrons (explicit calculations of this quantity are given in the following section).

\section{SOC MEDIATED SPIN TORQUE}

By definition, the spin torque $\mathbf{T}$ is the change of spin angular momentum per unit time. The average spin torque exerted by the itinerant electrons on the localized spins is then

$$
\mathbf{T}=\frac{d\langle\langle\mathbf{S}\rangle\rangle_{\mathrm{loc}}}{d t}=\frac{i}{\hbar}\left\langle\left\langle\left[H_{e x}^{\mathrm{loc}}, \mathbf{S}\right]\right\rangle\right\rangle_{\mathrm{loc}},
$$

where $\langle\langle\ldots\rangle\rangle_{\text {loc }}$ refers to the ensemble average over localized spins. Working out the commutator $\left[H_{e x}^{\text {loc }}, \mathbf{S}\right]$ and taking into account Eqs. (2), (5), and (8) we obtain

$$
\mathbf{T}=\frac{2 \Delta_{e x}}{\hbar^{2}}(\hat{\mathbf{M}} \times\langle\langle\mathbf{s}\rangle\rangle) .
$$

We note that, since $\langle\langle\mathbf{s}\rangle\rangle=(\hbar / 2)\langle\langle\boldsymbol{\sigma}\rangle\rangle$, Eq. (10) coincides (up to a factor $1 / M_{s}$ ) (Ref. 22) with Eq. (6) in Ref. 17.

When a current flows through the system, the spin density of the itinerant electrons deviates from its equilibrium value $\left(\langle\langle\mathbf{s}\rangle\rangle_{0}\right)$ by an amount $\langle\langle\delta \mathbf{s}\rangle\rangle$, i.e., $\langle\langle\mathbf{s}\rangle\rangle=\langle\langle\mathbf{s}\rangle\rangle_{0}+\langle\langle\delta \mathbf{s}\rangle\rangle$. In equilibrium there is no preferential direction for the motion of the itinerant electrons and, in average, the SOCF, which is an odd function of the momentum, vanishes. Thus the equilibrium spin density $\langle\langle\mathbf{s}\rangle\rangle_{0} \| \mathbf{M}$ and does not contribute to the spin torque. On the contrary, the presence of a current defines a preferential direction for the electron motion and results in a finite SOC mediated nonequilibrium spin density $\langle\langle\delta \mathbf{s}\rangle\rangle$, which is noncollinear with $\mathbf{M}$ and, in turn, induces a spin torque on the localized spins. The spin torque in Eq. (10) can then be rewritten as

$$
\mathbf{T}=\frac{2 \Delta_{e x}}{\hbar^{2}}(\hat{\mathbf{M}} \times\langle\langle\delta \mathbf{s}\rangle\rangle) .
$$

Considering Eq. (6) we find that the eigenenergies and wave functions of itinerant electrons are given, respectively, by

$$
E_{\mathbf{k}}^{ \pm}=\frac{\hbar^{2} k^{2}}{2 m} \pm\left|\mathbf{w}-\Delta_{e x} \hat{\mathbf{M}}\right|
$$

and

$$
\psi_{\mathbf{k}}^{ \pm}=\frac{e^{i \mathbf{k} \cdot \mathbf{r}}}{\sqrt{S\left(1+A_{ \pm}^{2}\right)}}\left(\begin{array}{c}
A_{ \pm} e^{i \xi_{\mathbf{k}}} \\
1
\end{array}\right)
$$

where $S$ is the area of the film,

$$
A_{ \pm}=\frac{\left(w_{z} M_{s}-\Delta_{e x} M_{z}\right) \pm M_{s}\left|\mathbf{w}-\Delta_{e x} \hat{\mathbf{M}}\right|}{\sqrt{\left(w_{x} M_{s}-\Delta_{e x} M_{x}\right)^{2}+\left(w_{y} M_{s}-\Delta_{e x} M_{y}\right)^{2}}},
$$

and

$$
\tan \xi_{\mathbf{k}}=-\frac{w_{y} M_{s}-\Delta_{e x} M_{y}}{w_{x} M_{s}-\Delta_{e x} M_{x}} .
$$

In writing Eqs. (14) and (15) we took into account that $|\mathbf{M}|$ $=M_{s}$.

The nonequilibrium spin density of itinerant electrons is determined by the relation

$$
\langle\langle\delta \mathbf{s}\rangle\rangle=\frac{\hbar}{2} \sum_{\sigma= \pm} \int \frac{d^{2} k}{(2 \pi)^{2}}\left\langle\Psi_{\mathbf{k}}^{\sigma}|\boldsymbol{\sigma}| \Psi_{\mathbf{k}}^{\sigma}\right\rangle \delta f_{\sigma}(\mathbf{k}),
$$

where $\delta f_{\sigma}=f_{\sigma}-f_{\sigma}^{(0)}$ represents the deviation of the distribution function $f_{\sigma}$ corresponding to the $\sigma$ band from its equilibrium value $f_{\sigma}^{(0)}$.

We consider the most relevant case (from the practical point of view) of a strong ferromagnet in which the exchange splitting dominates over the SOC effects and $E_{F} \gg \Delta_{e x}$ $\gg\left|\mathbf{w}\left(k_{F}\right)\right|$, where $k_{F}=\sqrt{2 m E_{F} / \hbar^{2}}$. In such a case the interband transitions can be neglected and the scattering by impurities 
can be treated within a constant relaxation-time approximation of the Boltzmann equation [for details see Refs. 16, 17, and 23]. One can then write ${ }^{24}$

$$
\delta f_{\sigma} \approx \frac{e \tau}{\hbar} \mathbf{E} \cdot \nabla_{\mathbf{k}} f_{\sigma}^{(0)},
$$

where $\tau$ is the relaxation time, $e$ is the electron charge ( $e$ $>0$ ), and $\mathbf{E}$ is the electric field.

The nonequilibrium spin density $\langle\langle\delta \mathbf{s}\rangle\rangle$ can be calculated by combining Eqs. (16) and (17). An analytical expression for $\langle\langle\delta \mathbf{s}\rangle\rangle$ can be obtained in the limit of large exchange coupling $\left(\Delta_{e x} \gg\left|\mathbf{w}\left(k_{F}\right)\right|\right)$ by expanding the spin expectation values $\left\langle\Psi_{\mathbf{k}}^{\sigma}|\boldsymbol{\sigma}| \Psi_{\mathbf{k}}^{\sigma}\right\rangle$ in powers of $w_{i} / \Delta_{e x}(i=x, y, z)$ and keeping up to the first order only. After integration over the inplane wave vector we obtain

$$
\begin{gathered}
\left\langle\left\langle\delta s_{x}\right\rangle\right\rangle=q \sum_{l=x, y} j_{l}\left[\left(M_{y}^{2}+M_{z}^{2}\right) c_{x}^{l}-M_{x} M_{y} c_{y}^{l}-M_{x} M_{z} c_{z}^{l}\right], \\
\left\langle\left\langle\delta s_{y}\right\rangle\right\rangle=q \sum_{l=x, y} j_{l}\left[-M_{x} M_{y} c_{x}^{l}+\left(M_{x}^{2}+M_{z}^{2}\right) c_{y}^{l}-M_{y} M_{z} c_{z}^{l}\right],
\end{gathered}
$$

and

$$
\left\langle\left\langle\delta s_{z}\right\rangle\right\rangle=q \sum_{l=x, y} j_{l}\left[-M_{x} M_{z} c_{x}^{l}-M_{y} M_{z} c_{y}^{l}+\left(M_{x}^{2}+M_{y}^{2}\right) c_{z}^{l}\right] .
$$

In the equations above $j_{l}$ represents the $l$ th component of the charge current density ${ }^{25} \mathbf{j}=\left(j_{x}, j_{y}, 0\right)^{T}$ and $q=m P / 2 e \Delta_{e x} M_{s}^{2}$, where $P$ is the spin polarization of the current.

By substituting Eqs. (18)-(20) into Eq. (11) and considering that $P \approx \Delta_{e x} / E_{F}$ we obtain the nonequilibrium spin torque,

$$
\mathbf{T}=-\frac{m \Delta_{e x}}{e \hbar^{2} E_{F} M_{s}}\left[j_{x}\left(\mathbf{M} \times \mathbf{d}_{x}\right)+j_{y}\left(\mathbf{M} \times \mathbf{d}_{y}\right)\right],
$$

where we have introduced the vectors

$$
\mathbf{d}_{l}=c_{x}^{l} \hat{\mathbf{x}}+c_{y}^{l} \hat{\mathbf{y}}+c_{z}^{l} \hat{\mathbf{z}} ; \quad(l=x, y),
$$

with $\hat{\mathbf{x}}, \hat{\mathbf{y}}$, and $\hat{\mathbf{z}}$ as the unit vectors along the $x, y$, and $z$ axes, respectively. Equation (21) is valid for strong ferromagnets and for linear in $\mathbf{k}$ but otherwise arbitrary SOCF and for any orientation of the magnetization. It reveals in an elegant and simple way how the nonequilibrium spin torque is determined by the SOCF whose properties are encoded in the vectors $\mathbf{d}_{l}$.

We remark that the SOC mediated spin torque in Eq. (21) is different from the conventional torque produced by injecting a spin-polarized current into a ferromagnetic layer. The conventional torque requires a source (usually an additional ferromagnetic layer) of spin-polarized electrons with noncollinear magnetization compared to the magnetization of the free layer (where the spin torque is exerted) and can be finite even in the absence of SOC. ${ }^{1-7}$ In contrast, the SOC mediated spin torque vanishes in the absence of SOC [see Eq. (21)] but does not require the injection of spin-polarized cur- rents and can, therefore, be present in structures containing a single ferromagnetic layer.

\section{LANDAU-LIFSHITZ-GILBERT EQUATION}

We now consider the presence of an external magnetic field and include the effects of the SOC mediated spin torque T [see Eq. (21)] in the standard LLG equation. ${ }^{26,27}$ The modified LLG equation describing the magnetization dynamics of a single ferromagnetic layer in the presence of SOC and subjected to an in-plane current flow and an external magnetic field reads

$$
\frac{d \mathbf{M}}{d t}=-\gamma_{0}\left(\mathbf{M} \times \mathbf{H}_{\mathrm{eff}}\right)+\frac{\alpha_{\mathrm{G}}}{M_{s}}\left(\mathbf{M} \times \frac{d \mathbf{M}}{d t}\right)+\gamma_{0} \mathbf{T},
$$

where $\alpha_{\mathrm{G}}$ is the Gilbert damping parameter and the effective field reads as

$$
\mathbf{H}_{\mathrm{eff}}=\mathbf{H}_{\mathrm{ext}}+\mathbf{H}_{\mathrm{an}}+\mathbf{H}_{\mathrm{d}}+A_{\mathrm{ex}} \nabla^{2} \mathbf{M} .
$$

Here $\mathbf{H}_{\text {ext }}$ is the applied external field, $\mathbf{H}_{\text {an }}$ is the anisotropy field, $\mathbf{H}_{\mathrm{d}}$ is the demagnetizing field due to axial dipole coupling, and $A_{\mathrm{ex}}$ is an exchange constant. ${ }^{27}$ Taking into account Eq. (21), one can rewrite the modified LLG equation [Eq. (23)] as

$$
\frac{d \mathbf{M}}{d t}=-\gamma_{0}\left[\mathbf{M} \times\left(\mathbf{H}_{\mathrm{eff}}+\mathbf{H}_{\mathrm{drive}}\right)\right]+\frac{\alpha_{\mathrm{G}}}{M_{s}}\left(\mathbf{M} \times \frac{d \mathbf{M}}{d t}\right),
$$

with the effective driving field

$$
\mathbf{H}_{\mathrm{drive}}=\frac{m \Delta_{e x}}{e \hbar^{2} E_{F} M_{s}}\left(j_{x} \mathbf{d}_{x}+j_{y} \mathbf{d}_{y}\right) .
$$

The SOC mediated driving field $\mathbf{H}_{\text {drive }}$ competes with $\mathbf{H}_{\text {eff }}$ [see Eq. (25)] and could be useful for current-driven magnetization switching in single, uniformly magnetized ferromagnetic layers, as already discussed in Refs. 16 and 17. However, since $\mathbf{H}_{\text {drive }}$ does not compete with the damping, the SOC mediated excitation of steady magnetization precessions in such systems is not possible.

\section{SOC MEDIATED SPIN TORQUE IN SYSTEMS WITH SIA AND/OR BIA}

In noncentrosymmetric materials, the lack of bulk inversion symmetry results in the BIA-induced SOC. ${ }^{28,29}$ This kind of SOC is, in general, present if the magnetic layer consists of a noncentrosymmetric ferromagnet and/or at interfaces between ferromagnets and noncentrosymmetric materials such as zinc blende semiconductors. ${ }^{30}$ The SIAinduced SOC does not need the presence of noncentrosymmetric materials; it originates from the lack of inversion symmetry of the structure itself (e.g., a ferromagnetic layer sandwiched between two different materials) and is determined by build-in and/or external electric fields. ${ }^{28,29}$ For systems with both BIA and SIA the two SOC mechanisms coexist. That is the case, for example, of zinc-blende semicondutor/cubic ferromagnet interfaces such as (001) 
TABLE I. SOC mediated spin torque in units of $m \Delta_{e x} / e \hbar^{2} E_{F} M_{s}$ for different growth directions.

\begin{tabular}{lcc}
\hline \hline Case & $\begin{array}{c}\text { Growth } \\
\text { direction }\end{array}$ & Spin torque \\
\hline A & $(001)$ & $j\left[\left(\alpha \sin \phi_{0}-\gamma \cos \phi_{0}\right)(\mathbf{M} \times \hat{\mathbf{x}})\right.$ \\
& & $\left.-\left(\alpha \cos \phi_{0}-\gamma \sin \phi_{0}\right)(\mathbf{M} \times \hat{\mathbf{y}})\right]$ \\
& & $j\left\{\alpha \sin \phi_{0}(\mathbf{M} \times \hat{\mathbf{x}})-\cos \phi_{0}[\beta(\mathbf{M} \times \hat{\mathbf{y}})\right.$ \\
B & $(110)$ & $+\lambda(\mathbf{M} \times \hat{\mathbf{z}})]\}$ \\
C & $(111)$ & $j(\alpha+\gamma)\left[\sin \phi_{0}(\mathbf{M} \times \hat{\mathbf{x}})-\cos \phi_{0}(\mathbf{M} \times \hat{\mathbf{y}})\right]$ \\
\hline \hline
\end{tabular}

$\mathrm{GaAs} / \mathrm{Fe}$, where the BIA-induced SOC of the noncentrosymmetric semiconductor interferes with the SIA-like SOC resulting from the strong build-in electric field at the interface. Such an interference leads to a net twofold symmetric SOCF which reflects the $C_{2 v}$ symmetry of the (001) GaAs/Fe interface. ${ }^{29,30}$

We focus now on the specific form of the nonequilibrium spin torque mediated by BIA- and SIA-induced SOCs in ferromagnetic layers grown in the directions (001), (110), and (111)

\section{A. (001) layers with axes $\hat{\mathbf{x}}\|[100], \hat{\mathbf{y}}\|[010]$, and $\hat{\mathbf{z}} \|[001]$}

The SOC containing both SIA-induced Bychkov-Rashba and BIA-induced Dresselhaus terms is given by ${ }^{18,19,29,31}$

$$
H_{\mathrm{SO}}=\alpha\left(k_{x} \sigma_{y}-k_{y} \sigma_{x}\right)+\gamma\left(k_{x} \sigma_{x}-k_{y} \sigma_{y}\right)
$$

where $\alpha$ and $\gamma$ are the corresponding Bychkov-Rashba and linearized-Dresselhaus parameters, respectively. The values of the SOC parameters are material dependent. For zincblende semiconductors the order of these parameters ranges from $10^{-3} \mathrm{eV} \AA$ to $10^{-1} \mathrm{eV} \AA{ }^{29}$ For the case of ferromagnetic materials the values of the SOC parameters are less known. The SOCFs in an Fe/GaAs slab have recently been extracted from $a b$ initio calculations. ${ }^{32}$ For such a structure the Bychkov-Rashba-type and Dresselhaus-type SOC parameters were found to vary from about $\pm 10^{-2}$ to $10^{-1} \mathrm{eV} \AA .^{32}$

The components of the SOCF, w, corresponding to Eq. (27) are $w_{x}=\gamma k_{x}-\alpha k_{y}, w_{y}=\alpha k_{x}-\gamma k_{y}$, and $w_{z}=0$. From Eqs. (7) and (22) one obtains

$$
\mathbf{d}_{x}=\gamma \hat{\mathbf{x}}+\alpha \hat{\mathbf{y}} ; \quad \mathbf{d}_{y}=-\alpha \hat{\mathbf{x}}-\gamma \hat{\mathbf{y}} .
$$

The SOC mediated spin torque can be straightforwardly obtained by placing the above relation into Eq. (21). The result is shown in Table I [see case A]. The angle $\phi_{0}$ denotes the direction of the in-plane charge current, i.e., $j_{x}=j \cos \phi_{0}$ and $j_{y}=j \sin \phi_{0}$. By introducing polar coordinates for the magnetization, $\mathbf{M}=M_{s}(\sin \theta \cos \phi, \sin \theta \sin \phi, \cos \theta)^{T}$, the components of the spin torque $\mathbf{T}=\left(T_{x}, T_{y}, T_{z}\right)^{T}$ can be rewritten as

$$
T_{x}=\frac{m \Delta_{e x} j}{e \hbar^{2} E_{F}}\left(\alpha \cos \phi_{0} \cos \theta-\gamma \sin \phi_{0} \cos \theta\right),
$$

$$
T_{y}=\frac{m \Delta_{e x} j}{e \hbar^{2} E_{F}}\left(\alpha \sin \phi_{0} \cos \theta-\gamma \cos \phi_{0} \cos \theta\right),
$$

and

$$
T_{z}=\frac{m \Delta_{e x} j}{e \hbar^{2} E_{F}} \sin \theta\left[\alpha \cos \left(\phi-\phi_{0}\right)-\gamma \sin \left(\phi+\phi_{0}\right)\right] .
$$

In the particular case of an in-plane magnetization (i.e., $\theta$ $=90^{\circ}$ ) we obtain, $T_{x}=T_{y}=0$ and

$$
\mathbf{T}=\frac{m \Delta_{e x} j}{e \hbar^{2} E_{F}}\left[\alpha \cos \left(\phi-\phi_{0}\right)-\gamma \sin \left(\phi+\phi_{0}\right)\right] \hat{\mathbf{z}} .
$$

After the transformations $\Delta_{e x} \rightarrow J_{s d}, \alpha \rightarrow-\alpha, \gamma \rightarrow \beta, \phi \rightarrow \theta$, and $\phi_{0} \rightarrow \theta_{0}$ so that the notations here and those used in Ref. 17 match each other, Eq. (32) reduces (up to a factor of 2) (Ref. 33) to Eq. (50) of Ref. 17.

\section{B. (110) layers with axes $\hat{\mathbf{x}}\|[1 \overline{10}], \hat{\mathbf{y}}\|[001]$, and $\hat{\mathbf{z}} \|[110]$}

Here the SOC is determined by $31,34,35$

$$
H_{\mathrm{SO}}=\beta k_{x} \sigma_{y}-\alpha k_{y} \sigma_{x}+\lambda k_{x} \sigma_{z},
$$

where $\alpha$ and $\beta$ are parameters related to the SIA-induced SOC and $\lambda$ is the strength of the BIA-induced SOC. Note that, in general, $\alpha \neq \beta$ which is a manifestation of the reduced symmetry of (110) layers with respect to the (001) structures. $^{31,34}$

The SOC mediated spin torque can be obtained by following the same procedure as before and the result is displayed as case B in Table I. In comparison with the previous case (case A) the spin torque posses now an extra component in the direction $\hat{\mathbf{z}} \times \mathbf{M}$.

\section{C. (111) layers with axes $\hat{\mathbf{x}}\|[11 \overline{2}], \hat{\mathbf{y}}\|[\overline{1} 10]$, and $\hat{z} \|[111]$}

In this case the SOC can be written as ${ }^{31,35}$

$$
H_{\mathrm{SO}}=(\alpha+\gamma)\left(k_{x} \sigma_{y}-k_{y} \sigma_{x}\right),
$$

where $\alpha$ and $\gamma$ are the strengths of the SIA- and BIA-induced SOCs, respectively. Equation (34) is equivalent to a Bychkov-Rashba SOC with strength $\alpha+\gamma$. Therefore, the spin torque can readily be obtained from the results in case A by performing the transformations $\gamma \rightarrow 0$ and $\alpha \rightarrow(\alpha+\gamma)$. The resulting spin torque is shown as case $\mathrm{C}$ in Table I. In the regime $\alpha=-\gamma$ (this could in principle be achieved by tuning $\alpha$ electrostatically) the SOC is zero [see Eq. (34)] and the spin torque vanishes. This open the possibility of switching the SOC mediated spin torque by means of a bias voltage.

\section{SPIN TORQUE MEDIATED BY STRAIN-INDUCED SOC}

We now investigate the effects of strain-induced SOC on the spin torque. We consider a (001) layer with axes $\hat{\mathbf{x}} \|[100]$, $\hat{\mathbf{y}} \|[010]$, and $\hat{\mathbf{z}} \|[001]$ with a strain-induced SOC of the form $^{36}$ 


$$
\begin{aligned}
H_{\mathrm{SO}}= & \alpha_{n}\left[\left(u_{z x} k_{z}-u_{x y} k_{y}\right) \sigma_{x}+\left(u_{x y} k_{x}-u_{y z} k_{z}\right) \sigma_{y}\right. \\
& \left.+\left(u_{y z} k_{y}-u_{z x} k_{x}\right) \sigma_{z}\right]+\gamma_{n}\left[k_{x}\left(u_{y y}-u_{z z}\right) \sigma_{x}\right. \\
& \left.+k_{y}\left(u_{z z}-u_{x x}\right) \sigma_{y}+k_{z}\left(u_{x x}-u_{y y}\right) \sigma_{z}\right],
\end{aligned}
$$

where $u_{i j}$ are the components of the strain tensor and $\alpha$ and $\gamma$ are material parameters. The spin torque deduced from Eq. (35) is given by

$$
\begin{aligned}
\mathbf{T}= & -\frac{m \Delta_{e x} j}{e \hbar^{2} E_{F} M_{s}}\left\{\alpha\left(u_{y z} \sin \phi_{0}-u_{z x} \cos \phi_{0}\right)(\mathbf{M} \times \hat{\mathbf{z}})\right. \\
& +\left[\alpha u_{x y} \cos \phi_{0}+\gamma\left(u_{z z}-u_{x x}\right) \sin \phi_{0}\right](\mathbf{M} \times \hat{\mathbf{y}}) \\
& \left.+\left[\gamma\left(u_{y y}-u_{z z}\right) \cos \phi_{0}-\alpha u_{x y} \sin \phi_{0}\right](\mathbf{M} \times \hat{\mathbf{x}})\right\} .
\end{aligned}
$$

The parametrical dependence of the SOC mediated, strain-induced spin torque is quite rich and suggests the possibility of engineering its form by appropriately designing the strain properties. In the particular case of an in-plane uniaxial strain such that the only nonvanishing components of the strain tensor are $u_{x x}=u_{y y} \neq u_{x y}=u_{y x}$ the spin torque acquires a form similar to the one given in case A but with strain-renormalized SIA and BIA parameters $\alpha \rightarrow \alpha u_{x y}$ and $\gamma \rightarrow \gamma u_{x x}$.

\section{SUMMARY}

We have derived a general expression for the currentinduced spin torque in a single ferromagnetic layer in the presence of a linear in momentum SOC field. We have performed explicit calculations of the spin torque in (001), (110), and (111) layers lacking bulk and/or structure inversion symmetry. The spin torque mediated by strain-induced SOC has also been investigated.

\section{ACKNOWLEDGMENTS}

We thank J. Fabian and M. Gmitra for valuable discussions. This work was supported by the Deutsche Forschungsgemeinschaft via Grant No. SFB 689 and the FONDECYT grant (Grant No. 1085229).
${ }^{1}$ L. Berger, Phys. Rev. B 54, 9353 (1996).

${ }^{2}$ J. Slonczewski, J. Magn. Magn. Mater. 159, L1 (1996).

${ }^{3}$ A. Brataas, Yu. V. Nazarov, and G. E. W. Bauer, Eur. Phys. J. B 22, 99 (2001).

${ }^{4}$ M. D. Stiles and A. Zangwill, Phys. Rev. B 66, 014407 (2002).

${ }^{5}$ J. Barnaś, A. Fert, M. Gmitra, I. Weymann, and V. K. Dugaev, Phys. Rev. B 72, 024426 (2005).

${ }^{6}$ A. Manchon, N. Ryzhanova, A. Vedyayev, M. Chschiev, and B. Dieny, J. Phys.: Condens. Matter 20, 145208 (2008).

${ }^{7}$ A. Slavin and V. Tiberkevich, IEEE Trans. Magn. 44, 1916 (2008).

${ }^{8}$ J. A. Katine, F. J. Albert, R. A. Buhrman, E. B. Myers, and D. C. Ralph, Phys. Rev. Lett. 84, 3149 (2000).

${ }^{9}$ M. Tsoi, J. Appl. Phys. 91, 6801 (2002).

${ }^{10}$ S. I. Kiselev, J. C. Sankey, I. N. Krivorotov, N. C. Emley, R. J. Schoelkopf, R. A. Buhrman, and D. C. Ralph, Nature (London) 425, 380 (2003).

${ }^{11}$ W. H. Rippard, M. R. Pufall, S. Kaka, S. E. Russek, and T. J. Silva, Phys. Rev. Lett. 92, 027201 (2004).

${ }^{12}$ S. M. Rezende, F. M. de Aguiar, and A. Azevedo, Phys. Rev. Lett. 94, 037202 (2005).

${ }^{13}$ M. Gmitra and J. Barnás, Phys. Rev. Lett. 96, 207205 (2006).

${ }^{14}$ D. C. Ralph and M. D. Stiles, J. Magn. Magn. Mater. 320, 1190 (2008).

${ }^{15}$ T. P. Pareek, Phys. Rev. B 75, 115308 (2007).

${ }^{16}$ A. Manchon and S. Zhang, Phys. Rev. B 78, 212405 (2008).

${ }^{17}$ A. Manchon and S. Zhang, Phys. Rev. B 79, 094422 (2009).

${ }^{18}$ Y. A. Bychkov and E. I. Rashba, J. Phys. C 17, 6039 (1984).

${ }^{19}$ G. Dresselhaus, Phys. Rev. 100, 580 (1955).

${ }^{20}$ M. B. Stearns, Phys. Rev. B 8, 4383 (1973).

${ }^{21}$ O. Johnson, Phys. Status Solidi B 99, 745 (1980).

${ }^{22}$ In the definition of the spin torque $\mathbf{T}[$ Eq. (6)] in Ref. 17 the authors use the magnetization $\mathbf{M}$ in place of the unit vector $\hat{\mathbf{M}}$ $=\mathbf{M} / M_{s}$. This leads to wrong units for $\mathbf{T}$. However, since the calculated torque [see, for example, Eq. (50) in Ref. 17] has correct units, the inappropriate replacement of $\hat{\mathbf{M}}$ by $\mathbf{M}$ in their Eq. (6) seems to be a misprint.

${ }^{23}$ J. Schliemann and D. Loss, Phys. Rev. B 68, 165311 (2003).

${ }^{24}$ An expression for $\delta f_{\sigma}$ which differs from Eq. (17) has been reported by M. Trushin and J. Schliemann [Phys. Rev. B 75, 155323 (2007)] for systems with Bychkov-Rashba and Dresselhaus SOCs. We found that, up to first order in the SOCF there is no difference between using our Eq. (17) or the expression given by Trushin and Schliemann. Similarly, the use of the velocity operator with or without SOC leads, up to first order in the SOCF, to the same results for the spin density [Eqs. (18) and (19)].

${ }^{25} \mathrm{Up}$ to first order in the SOCF the charge currents with and without SOC coincide.

${ }^{26}$ T. L. Gilbert, IEEE Trans. Magn. 40, 3443 (2004).

${ }^{27}$ Spin Dynamics in Confined Magnetic Structures I, Topics in Applied Physics, edited by B. Hillebrands and K. Ounadjela (Springer, New York, 2002), Vol. 83; Spin Dynamics in Confined Magnetic Structures II, Topics in Applied Physics (Springer, New York, 2003), Vol. 87.

${ }^{28}$ I. Žutić, J. Fabian, and S. Das Sarma, Rev. Mod. Phys. 76, 323 (2004).

${ }^{29}$ J. Fabian, A. Matos-Abiague, C. Ertler, P. Stano, and I. Žutić, Acta Phys. Slov. 57, 565 (2007).

${ }^{30}$ J. Moser, A. Matos-Abiague, D. Schuh, W. Wegscheider, J. Fabian, and D. Weiss, Phys. Rev. Lett. 99, 056601 (2007); A. Matos-Abiague and J. Fabian, Phys. Rev. B 79, 155303 (2009).

${ }^{31}$ X. Cartoixà, L.-W. Wang, D. Z.-Y. Ting, and Y.-C. Chang, Phys. Rev. B 73, 205341 (2006).

${ }^{32}$ M. Gmitra, A. Matos-Abiague, C. Ambrosch-Draxl, and J. Fabian, arXiv:0907.4149 (unpublished).

${ }^{33}$ The extra factor of 2 in Eq. (50) of Ref. 17 is a consequence of the same extra factor in Eq. (47) of that paper. A simple calcu- 
lation shows that this factor of 2 cannot be obtained when placing the wave functions [Eq. (37) in Ref. 17] into Eq. (47). We suspect the authors of Ref. 17 may have missed the cancellation of such a factor with the contribution $1 / 2$ from the normalization constant of the wave function.

${ }^{34}$ H. Diehl, V. A. Shalygin, V. V. Bel'kov, Ch. Hoffmann, S. N. Danilov, T. Herrle, S. A. Tarasenko, D. Schuh, Ch. Gerl, W.
Wegschelder, W. Prettl, and S. D. Ganichev, New J. Phys. 9, 349 (2007).

${ }^{35}$ A. Matos-Abiague, M. Gmitra, and J. Fabian, Phys. Rev. B 80, 045312 (2009).

${ }^{36}$ G. E. Pikus and A. N. Titkov, in Optical Orientation, Modern Problems in Condensed Matter Science, edited by F. Meier and B. P. Zakharchenya (North-Holland, Amsterdam, 1984), Vol. 8. 